\title{
Structural basis for C-type inactivation in a Shaker family voltage gated $\mathrm{K}^{+}$channel
}

Ravikumar Reddi', Kimberly Matulef ${ }^{1}$, Erika A. Riederer ${ }^{1}$, Matthew R. Whorton ${ }^{2}$ and

Francis I. Valiyaveetil ${ }^{*}$

From the ${ }^{1}$ Program in Chemical Biology

Department of Chemical Physiology and Biochemistry

${ }^{2}$ Vollum Institute

Oregon Health \& Science University

3181 SW Sam Jackson Park Rd

Portland, OR 97239

*Correspondence to FIV; valiyave@ohsu.edu 


\author{
Abstract: \\ C-type inactivation is a process by which ion flux through a voltage-gated $\mathrm{K}^{+}\left(\mathrm{K}_{\mathrm{v}}\right)$ channel is \\ regulated at the selectivity filter. While prior studies have indicated that $C$-type inactivation \\ involves structural changes at the selectivity filter, the nature of the changes have not been \\ resolved. Here we report the crystal structure of the $\mathrm{K}_{\mathrm{v}} 1.2$ channel in a C-type inactivated state. \\ The structure shows that $\mathrm{C}$-type inactivation involves changes in the selectivity filter that disrupt \\ the outer two ion binding sites in the filter. The changes at the selectivity filter propagate to the \\ extracellular mouth and the turret regions of the channel pore. The structural changes observed \\ are consistent with the functional hallmarks of C-type inactivation. This study highlights the \\ intricate interplay between $\mathrm{K}^{+}$occupancy at the ion binding sites and the interactions of the \\ selectivity filter in determining the balance between the conductive and the inactivated \\ conformations of the filter.
}




\section{Introduction:}

Voltage gated $\mathrm{K}^{+}$channels $\left(\mathrm{K}_{\mathrm{v}}\right)$ are essential for the generation and conduction of electrical signals by neurons, muscle and endocrine cells.(1) $\mathrm{K}_{\mathrm{v}}$ channels are tetrameric proteins that contain a central pore domain and peripheral voltage sensor domains (VSD, Fig.1a).(2, 3) The pore domain houses the pathway for $\mathrm{K}^{+}$ions across the membrane (Fig. 1b). The activation of a $\mathrm{K}_{\mathrm{v}}$ channel takes place upon membrane depolarization and involves an outward movement of the VSDs.(4) This movement of the VSD is coupled to the opening of pore domain to turn on the $\mathrm{K}^{+}$flux across the membrane. On sustained activation, the flux of $\mathrm{K}^{+}$ through the $\mathrm{K}_{\mathrm{v}}$ channel is turned off through mechanisms that are referred to as inactivation.(5)

In the Shaker family of $\mathrm{K}_{\mathrm{v}}$ channels, there are two types of inactivation, $\mathrm{N}$-type and Ctype. $(6,7)$ In $\mathrm{N}$-type inactivation, the $\mathrm{N}$-terminus of the channel binds to the open pore domain and occludes the ion pathway. $(6,8)$ Shaker channels with the N-terminal deleted do not undergo $\mathrm{N}$-type inactivation but inactivate through a different mechanism called C-type inactivation.(7) This mechanism is also referred to as slow inactivation as the timescale is generally slower, on the order of seconds, compared to the millisecond time scale observed for $\mathrm{N}$-type inactivation.(9) C-type inactivation is a physiologically important process that can regulate cell excitability by determining channel availability. $(10,11)$

The mechanism of C-type inactivation has been investigated using functional, spectroscopic, structural and computational approaches.(5) All these approaches suggest that C-type inactivation involves changes at the selectivity filter. The selectivity filter refers to the narrow region of the ion pathway in the pore domain, where selection for $\mathrm{K}^{+}$takes place (Fig. 1c).(12) The selectivity filter consists of a row of $\mathrm{K}^{+}$binding sites (called S1- S4 extracellular intracellular) that are constructed from the main chain carbonyl atoms and the threonine side chain from the protein sequence T-V-G-Y-G.(13) The structures of the selectivity filter of $\mathrm{K}^{+}$ channels are highly conserved. The structures determined mainly show the selectivity filter in a conductive state, the state of the selectivity filter that supports the flux of $\mathrm{K}^{+}$through the channel. During C-type inactivation, there are structural changes at the selectivity filter that convert the selectivity filter from a conductive to a non-conductive conformation. $(5,9)$

Understanding the mechanism of C-type inactivation requires the structure of the selectivity filter in the C-type inactivated state. The structure of a non-conducting mutant of the $\mathrm{K}_{\mathrm{v}} 1.2$ channel has been proposed to show the selectivity filter in the C-type inactivated state.(14) However, the structural changes observed were minimal, which raises a question on 
whether this structure truly represents the C-type inactivated state.(15) Other structural studies on C-type inactivation have used the $\mathrm{K}^{+}$channel $\mathrm{KcsA}$. KcsA does not belong to the $\mathrm{K}_{\mathrm{v}}$ family but undergoes an inactivation process that bears some functional similarity to C-type inactivation in $\mathrm{K}_{\mathrm{v}}$ channels.(16) Structural studies on the KcsA channel under conditions that favor inactivation show a constriction of the selectivity filter. $(17,18)$ However, the relevance of this constricted conformation to $\mathrm{C}$-type inactivation in $\mathrm{K}_{\mathrm{v}}$ channels has been debated.(19)

Here we report the crystal structure of the $\mathrm{K}_{\mathrm{v}} 1.2$ channel in the C-type inactivated state. We carry out structural studies on a mutant of the $\mathrm{K}_{\mathrm{v}} 1.2$ channel that shows a vastly increased rate of $\mathrm{C}$-type inactivation. We observe that $\mathrm{C}$-type inactivation involves a dilation of the outer ion binding sites of the selectivity filter along with changes in the extracellular mouth and the turret regions of the pore domain. Our studies highlight the selectivity filter interactions that are important for C-type inactivation and suggest a molecular pathway for this process.

\section{Results:}

\section{C-type inactivation in the $K_{v} 1.2-2.1$ channel.}

Our strategy for determining the structure of the $\mathrm{K}_{\mathrm{v}} 1.2$ channel in the C-type inactivated state was to use mutants of this channel that show an enhanced rate of inactivation with the expectation that these mutants will trap the selectivity filter in the C-type inactivated state. In the Shaker channel, the W434F substitution dramatically increases the rate of inactivation and this substitution has been extensively used in functional studies to mimic the C-type inactivated state.(20) The corresponding mutation, W366F in the $\mathrm{K}_{v} 1.2$ channel has been shown to have an increased rate of inactivation (Supplementary fig. 1).(21, 22) For our studies, we used a variant of the $\mathrm{K}_{\mathrm{v}} 1.2$ channel referred to as the $\mathrm{K}_{\mathrm{v}} 1.2-2.1$ chimera (referred henceforth as $\mathrm{K}_{\mathrm{v}} 1.2-$ 2.1) as this construct affords crystals that diffract to a higher resolution.(3) In the $K_{v} 1.2-2.1$ channel, residues 267-302 in the S3-S4 loop in the voltage sensor domain are substituted by residues 274-305 of the $\mathrm{K}_{\mathrm{v}} 2.1$ channel. The $\mathrm{K}_{\mathrm{v}} 1.2-2.1$ channel shows similar functional properties to the $\mathrm{K}_{v} 1.2$ channel and the W362F (equivalent to W366F in $\mathrm{K}_{v} 1.2$ ) substitution in the $K_{v} 1.2-2.1$ channel shows a similar enhancement in the rate of inactivation (Fig. 1d).(23)

Our attempts at structural studies of the $\mathrm{K}_{\mathrm{v}} 1.2-2.1-\mathrm{W} 362 \mathrm{~F}$ channel were stymied as the channel showed poor expression and suboptimal biochemistry (Fig. 1e). We therefore screened for additional amino acid substitutions that improved protein expression and 
biochemical behavior while maintaining an enhanced rate of C-type inactivation. These efforts identified a construct with two additional substitutions, S367T and V377T, which exhibited good biochemical behavior and an enhanced rate of inactivation (Fig. 1d, e). We refer to the $\mathrm{K}_{\mathrm{v}} 1.2-$ 2.1 channel with the W362F, S367T and V377T substitutions as the $\mathrm{K}_{\mathrm{v}}$ 1.2-2.1-3m channel. At high $\mathrm{K}^{+}$, the $\mathrm{K}_{\mathrm{v}} 1.2-2.1-3 \mathrm{~m}$ channel showed a 2000 -fold increase in the rate of C-type inactivation while at low external $\mathrm{K}^{+}$, the rate of $\mathrm{C}$-type inactivation was very fast and could not be accurately determined. Based on the rapid rate of inactivation, we anticipated that the crystal structure of the $\mathrm{K}_{\mathrm{v}} 1.2-2.1-3 \mathrm{~m}$ channel would reveal the selectivity filter in the C-type inactivated state.

Structure of the $K_{v}$ 1.2-2.1-3m channel. For structural studies, the $K_{v} 1.2-2.1-3 m$ channel was co-expressed with the beta subunit and the channel-beta complex was crystallized in the presence of $150 \mathrm{mM} \mathrm{K}^{+}$. We obtained crystals of the same space group $\left(P 42{ }_{1} 2\right)$ as reported for the $\mathrm{K}_{\mathrm{v}}$ 1.2-2.1 channel but with slightly altered unit cell parameters.(3) Data from multiple crystals were combined to obtain a complete data set to $3.35 \AA$ resolution and the structure was determined by molecular replacement using the $\mathrm{K}_{\mathrm{v}}$ 1.2-2.1 (pdb: $2 \mathrm{r} 9 \mathrm{r}$ ) channel structure as the model (Supplementary table 1). There are two copies (molecule I and II) of the channel-beta complex in the asymmetric unit. The beta subunit and the T1 domain in both molecules were well resolved while the transmembrane region in molecule I was better resolved than in molecule II. For the transmembrane region, we observed stronger electron density for the pore domain compared to the voltage sensor. Within the pore domain, the electron density for Y373 -D375 was weaker compared to the neighboring residues and electron density for the carbonyl group for the G374 residue was not observed. Electron density corresponding to the pore helix and the selectivity filter are shown in figure $2 a, b$. In the voltage sensor domain, the electron density was sufficient for modelling the transmembrane segments (Supplementary fig. 2) while electron density for the loops connecting the TM segments and the linker connecting the T1 domain to the first transmembrane segment were not observed and these regions were therefore not modelled. A superposition of the various domains in the $K_{v} 1.2-2.1-3 m$ channel to $\mathrm{K}_{\mathrm{v}}$ 1.2-2.1 channel shows that the structural changes observed are mainly in the selectivity filter region and in the extracellular mouth of the pore (Supplementary fig. 3). 
The selectivity filter in the $K_{v} 1.2-2.1-3 m$ channel. A comparison of the selectivity filter in $\mathrm{K}_{\mathrm{v}} 1.2-2.1-3 \mathrm{~m}$ to the wild type channel shows substantial changes in the sidechain and backbone conformations (Fig. 2c, d). A major change observed is a reorientation of the Tyr side chain (Fig. 2e, f). In the $\mathrm{K}_{\mathrm{v}}$ 1.2-2.1 channel, the Tyr side chain interacts with the Trp363 and Ser 367 of the adjacent subunit while in the $\mathrm{K}_{\mathrm{v}} 1.2-2.1-3 \mathrm{~m}$ channel, these $\mathrm{H}$-bond interactions are broken and the Tyr side chain undergoes a $77^{\circ}$ rotation and the hydroxyl group in the Tyr side chain is now oriented towards the extracellular surface.

Another major change observed in the $\mathrm{K}_{\mathrm{v}} 1.2-2.1-3 \mathrm{~m}$ channel is a flip of the Asp375 side chain (Fig. 2g, h). In the $\mathrm{K}_{\mathrm{v}} 1.2-2.1$ structure, Asp375 forms an $\mathrm{H}$-bond with Trp362 in the pore helix. This $\mathrm{H}$-bond is disrupted in the $\mathrm{K}_{\mathrm{v}} 1.2-2.1-3 \mathrm{~m}$ channel due to the W362F substitution. The Asp375 side chain is now oriented towards the extracellular solution and forms a $\mathrm{H}$-bond with Thr377 in the adjacent subunit (distance of 3.32 $\AA$ ). The $K_{v} 1.2-2.1$ channel has a Val at this position, which is substituted by Thr in the $K_{v} 1.2-2.1-3 m$ channel. The flip of the Asp side chain as observed changes the side chain from being oriented towards the channel interior (in $\mathrm{K}_{v} 1.2-$ 2.1 ) to facing the extracellular solution.

The changes in the Tyr373 and Asp375 side chain conformations cause a widening of the selectivity filter towards the extracellular side. Due to this widening, the S1 and S2 ion binding sites are disrupted and instead form a vestibule (Fig. $2 \mathrm{c}, \mathrm{d}$ ). In $\mathrm{K}_{\mathrm{v}} 1.2-2.1$, $\mathrm{K}^{+}$binding is observed at the $\mathrm{S} 1$ and the $\mathrm{S} 2$ sites while in the $\mathrm{K}_{\mathrm{v}} 1.2-2.1-3 \mathrm{~m}$ channel only a weak electron density is observed in this vestibule region (Fig. $2 b$, Supplementary fig. 4). The electron density in vestibule region can correspond either to a low occupancy $\mathrm{K}^{+}$ion or a water molecule. The distances between the carbonyl oxygens in the vestibule region are however too large to tightly coordinate either a water or $\mathrm{a} \mathrm{K}^{+}$ion. In contrast to the changes seen at the $\mathrm{S} 1$ and the S2 sites, the $S 3$ and the $S 4$ sites in the $K_{v} 1.2-2.1-3 m$ filter are well superimposed on the corresponding sites in the $\mathrm{K}_{\mathrm{v}} 1.2-2.1$ filter and electron density corresponding to ion binding at these sites is observed. Previous studies have implicated the outer sites in the selectivity filter in C-type inactivation $(24,25)$ and the $\mathrm{K}_{\mathrm{v}} 1.2-2.1-3 \mathrm{~m}$ structure indicates that inactivation involves a disruption of the $\mathrm{S} 1$ and the $\mathrm{S} 2$ sites in the selectivity filter. A key characteristic of $\mathrm{C}$-type inactivation is a dependence on the extracellular $\mathrm{K}^{+}$concentration. $(26,27)$ The structure suggests that extracellular $\mathrm{K}^{+}$is protective for the filter against $\mathrm{C}$-type inactivation as higher extracellular $\mathrm{K}^{+}$concentration will increase the ion occupancy at the S1/S2 sites to prevent the dilation of these sites. 
Changes in the extracellular mouth of the $K_{v} 1.2-2.1-3 m$ pore. The changes in the selectivity filter in the $\mathrm{K}_{\mathrm{v}} 1.2-2.1-3 \mathrm{~m}$ structure are propagated to the loop region linking the selectivity filter to TM6 (Fig. 3a). The changes observed in this region are mainly due to the repositioning of the Asp375 side chain (Fig. 3b,c). Studies on the Shaker $\mathrm{K}^{+}$channel have suggested that residues corresponding to 376-378 in the $\mathrm{K}_{\mathrm{v}} 1.2-2.1-3 \mathrm{~m}$ channel change accessibility on C-type inactivation.(28) Studies on Shaker have also shown that a Cys substitution at 448 (376 in $\mathrm{K}_{\mathrm{v}}$ 1.2-2.1-3m) exhibits an enhanced rate of formation of a disulfide bond while a Cys substitution at 449 (377 in $\left.\mathrm{K}_{\mathrm{v}} 1.2-2.1-3 \mathrm{~m}\right)$ can form a high affinity metal $\left(\mathrm{Cd}^{2+}\right.$ or $\left.\mathrm{Zn}^{2+}\right)$ binding site in the inactivated state. $(28,29)$ Comparison of the $376-378$ region in the $\mathrm{K}_{\mathrm{v}} 1.2-2.1-3 \mathrm{~m}$ to the $K_{v} 1.2-2.1$ shows an increase in surface exposure of these residues (Supplementary fig. 5), which may underlie the changes in modification observed (for Cys substitutions at these sites) on C-type inactivation. In the $\mathrm{K}_{\mathrm{v}} 1.2-2.1-3 \mathrm{~m}$ structure, the $\mathrm{C} \alpha-\mathrm{C} \alpha$ distance between the 376 sidechains in the adjacent subunits is similar to the distance observed in the $\mathrm{K}_{\mathrm{v}} 1.2-2.1$ structure (Fig. 3b, c). The $K_{v} 1.2-2.1-3 m$ structure therefore does not account for the enhanced rate of disulfide bond formation by Cys 376 in the C-type inactivated state. Similarly the change in the $\mathrm{C} \alpha$ - $\mathrm{C} \alpha$ distance of the 377 sidechains in these structures is $0.51 \AA$ (14.06 to $13.55 \AA$ ), and this distance is still longer than expected for the formation of a metal coordination site by the Cys 377 sidechains from adjacent subunits.(30) The $\mathrm{K}_{\mathrm{v}}$ 1.2-2.1-3m channel structure therefore does not directly account for some of the changes in the extracellular mouth of the pore on C-type inactivation as inferred from the functional studies. One possibility is that the full extent of changes in the extracellular mouth of the pore on C-type inactivation are not revealed in the $\mathrm{K}_{\mathrm{v}} 1.2-2.1-3 \mathrm{~m}$ structure. Another possibility is that these experimental findings reflect greater flexibility in the extracellular mouth of the channel in the C-type inactivated state. An increased flexibility in the extracellular mouth on C-type inactivation could explain the poor electron density observed in the Y373-D375 region in the $\mathrm{K}_{\mathrm{v}} 1.2-2.1-3 \mathrm{~m}$ channel.

C-type inactivation also involves changes in the turret region present between TM5 and the pore helix. Fluorescent probes introduced into the turret region show a change in fluorescence on C-type inactivation.(31-33) We see structural changes in the turret region of the $\mathrm{K}_{\mathrm{v}} 1.2-2.1-3 \mathrm{~m}$ corresponding to the sites of introduction of the fluorescence probes (Fig. 3a). A key interaction that is altered in the $\mathrm{K}_{\mathrm{v}} 1.2-2.1-3 \mathrm{~m}$ channel is that of the E346 residue, which is towards the top of TM5 (Fig. 3d, e). Mutations at this Glu (E418 in the Shaker channel) increase the rate of inactivation. $(33,34)$ In the $\mathrm{K}_{\mathrm{v}} 1.2-2.1$ channel, E346 interacts with the poreS6 loop region of the channel through $\mathrm{H}$-bond interactions with the protein backbone of Thr379 
and the side chain of Thr380. In the $\mathrm{K}_{\mathrm{v}} 1.2-2.1-3 \mathrm{~m}$ structure, we observe a change in the E346 side chain conformation and correspondingly the interactions of the E346 side chain with the T379-T380 residues are altered.

\section{Structure of the $K_{v} 1.2-2.1-W 362 F$, S367T channel.}

To test the importance of the interaction between Asp375 and Thr377 for C-type inactivation, we investigated the functional properties of $a K_{v} 1.2-2.1$ channel with the native Val at 377 along with the W362F and S367T substitution (referred to as $\mathrm{K}_{\mathrm{v}} 1.2-2.1-2 \mathrm{~m}$ ). We observed that the inactivation was slow in this mutant compared to the very rapid inactivation observed in the $\mathrm{K}_{\mathrm{v}} 1.2-2.1-3 \mathrm{~m}$ channel. Inactivation in the $\mathrm{K}_{\mathrm{v}} 1.2-2.1-2 \mathrm{~m}$ channel was similar to the inactivation observed in the Kv1.2-2.1 channel (Fig. 4a). We crystallized and determined the structure of the $\mathrm{K}_{\mathrm{v}} 1.2-2.1-2 \mathrm{~m}$ channel at $150 \mathrm{mM} \mathrm{K}^{+}$(Fig. 4b, Supplementary table 1). In the $\mathrm{K}_{\mathrm{v}}$ 1.2-2.1-2m structure, we observed the selectivity filter in a conductive conformation as anticipated based on the similarity of the inactivation properties of the $K_{v} 1.2-2.1-2 m$ to the $K_{v} 1.2-$ 2.1 channel (Fig. 4c). These findings suggest a role for the inter-subunit Asp375-Thr377 interaction in stabilizing the selectivity filter in the C-type inactivated state. The equivalent position in the Shaker channel (Thr449) is an important locus for C-type inactivation and substitution of T449 in the Shaker channel with Val results in a non-inactivating phenotype.(26) Our studies suggests that substitutions at this site affect C-type inactivation through an effect on the interaction with the Asp residue.

\section{Discussion:}

Here we report the structure of the $\mathrm{K}_{\mathrm{v}} 1.2$ channel in the C-type inactivated state (Fig. 5a). The structure shows that the C-type inactivation involves a dilation of the selectivity filter at the S1 and S2 ion binding sites, which is caused by structural changes at the conserved Tyr and Asp side chains. The disruption of the $\mathrm{S} 1$ and the $\mathrm{S} 2$ ion binding sites of the filter perturbs $\mathrm{K}^{+}$ flux through the channel. The structure shows that the changes in the selectivity filter on C-type inactivation are propagated to the extracellular mouth and the turret region of the pore domain of the channel. The structure of the selectivity filter in the C-type inactivated state is consistent with the dilation model for inactivation previously proposed by Hoshi and Armstrong.(9) 
Based on our structural studies, we propose the following transitions at the selectivity filter during C-type inactivation (Fig. 5b). The process initiates with a loss of ion occupancy at the S1 and S2 sites in the selectivity filter. The loss of ion occupancy causes a flip of 1' and the 2' carbonyl groups, which is accompanied by a rotation of the Tyr373 side chain. The movement of the Tyr side chain sets up a clash with the Asp375 sidechain and as a result, the Asp375-Trp362 $\mathrm{H}$ bond interaction is broken and the Asp side chain switches to facing the extracellular side. Asp375 in this conformation is stabilized by an interaction with Thr377. This change in the conformation of Asp375 flips the 0' carbonyl group. The changes in the 0'-2' carbonyl groups result in a dilation of the S1 and the S2 sites in the selectivity filter, which prevents ion coordination at these sites and thereby affects ion conduction through the channel. The changes in the Tyr and the Asp side chain also change the surface accessibility and dynamics in the filter-TM6 region. These changes are also transmitted to the pore turret through changes in the interaction of E346 with the filter-TM6 loop. Through this process, structural changes that are initiated by changes in ion occupancy at the selectivity filter are propagated throughout the selectivity filter and the extracellular mouth of the pore.

A structure of the $\mathrm{K}_{\mathrm{v}} 1.2-2.1-\mathrm{V} 406 \mathrm{~W}$ channel has been reported as presenting the selectivity filter in the C-type inactivated state (Supplementary fig. 6).(14) The structural change observed in the selectivity filter, compared to the conductive state, is a small distortion of the S1 site.(15) This is in contrast to the abrogation of the $S 1$ and $S 2$ sites observed in the $K_{v} 1.2-2.1$ $3 \mathrm{~m}$ structure. The proposed inactivated state in the KcsA channel shows a distortion of the S2 and the S3 sites of the selectivity filter and is distinct from the structure of the selectivity filter in the $\mathrm{K}_{\mathrm{v}}$ 1.2-2.1-3m channel (Supplementary fig. 6). Gating of $\mathrm{K}^{+}$flux through two-pore $\mathrm{K}^{+}(\mathrm{K} 2 \mathrm{P})$ channels takes place at the selectivity filter by a mechanism that shares a functional resemblance to C-type inactivation in $\mathrm{K}_{\mathrm{v}}$ channels.(35) A recent structural study on the TREK-1 $\mathrm{K} 2 \mathrm{P}$ channel suggested that the gating process involves a loss of the $\mathrm{S} 1$ and the $\mathrm{S} 2$ ion binding sites in the selectivity filter while a structure of the TASK 2-P K $\mathrm{K}^{+}$channel indicated a loss of the S1 (and potentially the S0) binding site in the inactivated state.(36, 37) These structures suggest that $\mathrm{K}^{+}$channels, which have essentially identical selectivity filters, can have distinct mechanisms of inactivation or gating at the selectivity filter.

C-type inactivation is widespread in the $\mathrm{K}_{\mathrm{v}}$ channel family with the inactivation properties that are tuned for the specific physiological roles of these channels. Structural studies of these other $\mathrm{K}_{\mathrm{v}}$ family members will be necessary to determine whether conformational changes similar 
to that observed in the $\mathrm{K}_{\mathrm{v}} 1.2-2.1-3 \mathrm{~m}$ structure underlie inactivation or gating at the selectivity filter in diverse $K_{v}$ channels.

\section{Acknowledgements:}

We thank Dr. Roderick Mackinnon for providing the $\mathrm{K}_{\mathrm{v}} 1.2-2.1 /$ beta plasmid. We thank Dr. Shivani Ahuja and Dr. Kim Hartfield for help with protein expression in Pichia, protein purification and analysis. We thank Dr. Eric Gouaux for providing access to crystallization equipment. Crystallography data were collected at GM/CA beamlines 23ID-B and 23ID-D at the Advanced Photon Source at Argonne National Laboratory and we thank the staff at the beamlines for their support with data collection. GM/CA @ APS has been funded in whole or in part with Federal funds from the National Cancer Institute (Y1-CO-1020) and the National Institute of General Medical Science (Y1-GM-1104). Use of the Advanced Photon Source was supported by the U.S. Department of Energy, Basic Energy Sciences, Office of Science, under contract No. W-31-109-ENG-38. This research was supported by a grant from the National Institute of General Medical Sciences (R01GM087546) of the National Institutes of Health to FIV. EAR was supported by a pre-doctoral fellowship from the American Heart Association (AHA 19PRE34380950).

\section{References:}

1. B. Hille, Ion Channels of Excitable Membranes (Sinauer Sunderland, MA, 2001).

2. S. B. Long, E. B. Campbell, R. Mackinnon, Crystal structure of a mammalian voltagedependent Shaker family K+ channel. Science 309, 897-903 (2005).

3. S. B. Long, X. Tao, E. B. Campbell, R. MacKinnon, Atomic structure of a voltagedependent K+ channel in a lipid membrane-like environment. Nature 450, 376-382 (2007).

4. D. M. Kim, C. M. Nimigean, Voltage-Gated Potassium Channels: A Structural Examination of Selectivity and Gating. Cold Spring Harb Perspect Biol 8 (2016).

5. H. T. Kurata, D. Fedida, A structural interpretation of voltage-gated potassium channel inactivation. Prog Biophys Mol Biol 92, 185-208 (2006).

6. T. Hoshi, W. N. Zagotta, R. W. Aldrich, Biophysical and molecular mechanisms of Shaker potassium channel inactivation. Science. 250, 533-538. (1990).

7. T. Hoshi, W. N. Zagotta, R. W. Aldrich, Two types of inactivation in Shaker K+ channels: effects of alterations in the carboxy-terminal region. Neuron 7, 547-556 (1991).

8. M. Zhou, J. Morais-Cabral, S. Mann, R. MacKinnon, Potassium channel receptor site for the inactivation gate and quaternary amine inhibitors. Nature 411, 657-661 (2001).

9. T. Hoshi, C. M. Armstrong, C-type inactivation of voltage-gated K+ channels: pore constriction or dilation? J Gen Physiol 141, 151-160 (2013). 
10. R. W. Aldrich, Jr., P. A. Getting, S. H. Thompson, Mechanism of frequency-dependent broadening of molluscan neurone soma spikes. J Physiol 291, 531-544 (1979).

11. J. Roeper, C. Lorra, O. Pongs, Frequency-dependent inactivation of mammalian A-type $\mathrm{K}+$ channel KV1.4 regulated by Ca2+/calmodulin-dependent protein kinase. J Neurosci 17, 3379-3391 (1997).

12. R. MacKinnon, Nobel Lecture. Potassium channels and the atomic basis of selective ion conduction. Biosci Rep 24, 75-100 (2004).

13. Y. Zhou, J. H. Morais-Cabral, A. Kaufman, R. MacKinnon, Chemistry of ion coordination and hydration revealed by a $\mathrm{K}+$ channel-Fab complex at $2.0 \mathrm{~A}$ resolution. Nature $\mathbf{4 1 4}$, 43-48 (2001).

14. V. Pau, Y. Zhou, Y. Ramu, Y. Xu, Z. Lu, Crystal structure of an inactivated mutant mammalian voltage-gated K(+) channel. Nat Struct Mol Biol 24, 857-865 (2017).

15. F. I. Valiyaveetil, A glimpse into the C-type-inactivated state for a Potassium Channel. Nat Struct Mol Biol 24, 787-788 (2017).

16. J. F. Cordero-Morales, L. G. Cuello, E. Perozo, Voltage-dependent gating at the KcsA selectivity filter. Nat Struct Mol Biol 13, 319-322 (2006).

17. L. G. Cuello, V. Jogini, D. M. Cortes, E. Perozo, Structural mechanism of C-type inactivation in $\mathrm{K}(+)$ channels. Nature 466, 203-208 (2010).

18. L. G. Cuello, D. M. Cortes, E. Perozo, The gating cycle of a $\mathrm{K}(+)$ channel at atomic resolution. Elife 6 (2017).

19. P. K. Devaraneni et al., Semisynthetic K+ channels show that the constricted conformation of the selectivity filter is not the C-type inactivated state. Proc Natl Acad Sci U S A 110, 15698-15703 (2013).

20. Y. Yang, Y. Yan, F. J. Sigworth, How does the W434F mutation block current in Shaker potassium channels? J Gen Physiol 109, 779-789 (1997).

21. J. F. Cordero-Morales, V. Jogini, S. Chakrapani, E. Perozo, A multipoint hydrogen-bond network underlying KcsA C-type inactivation. Biophys J 100, 2387-2393 (2011).

22. E. Suarez-Delgado et al., KV1.2 channels inactivate through a mechanism similar to Ctype inactivation. J Gen Physiol 152 (2020).

23. X. Tao, R. MacKinnon, Functional analysis of Kv1.2 and paddle chimera Kv channels in planar lipid bilayers. J Mol Biol 382, 24-33 (2008).

24. T. Baukrowitz, G. Yellen, Use-dependent blockers and exit rate of the last ion from the multi-ion pore of a K+ channel. Science 271, 653-656 (1996).

25. L. Kiss, S. J. Korn, Modulation of C-type inactivation by $\mathrm{K}+$ at the potassium channel selectivity filter. Biophys J 74, 1840-1849 (1998).

26. J. Lopez-Barneo, T. Hoshi, S. H. Heinemann, R. W. Aldrich, Effects of external cations and mutations in the pore region on C-type inactivation of Shaker potassium channels. Receptors Channels 1, 61-71 (1993).

27. T. Baukrowitz, G. Yellen, Modulation of $\mathrm{K}+$ current by frequency and external $[\mathrm{K}+]$ : a tale of two inactivation mechanisms. Neuron 15, 951-960 (1995).

28. Y. Liu, M. E. Jurman, G. Yellen, Dynamic rearrangement of the outer mouth of a $\mathrm{K}_{+}$ channel during gating. Neuron 16, 859-867 (1996). 
29. G. Yellen, D. Sodickson, T. Y. Chen, M. E. Jurman, An engineered cysteine in the external mouth of a $\mathrm{K}+$ channel allows inactivation to be modulated by metal binding. Biophys J 66, 1068-1075 (1994).

30. H. S. Krovetz, H. M. VanDongen, A. M. VanDongen, Atomic distance estimates from disulfides and high-affinity metal-binding sites in a K+ channel pore. Biophys J 72, 117126 (1997).

31. A. Cha, F. Bezanilla, Characterizing voltage-dependent conformational changes in the Shaker K+ channel with fluorescence. Neuron 19, 1127-1140 (1997).

32. C. S. Gandhi, E. Loots, E. Y. Isacoff, Reconstructing voltage sensor-pore interaction from a fluorescence scan of a voltage-gated K+ channel. Neuron 27, 585-595 (2000).

33. E. Loots, E. Y. Isacoff, Molecular coupling of $\mathrm{S} 4$ to a $\mathrm{K}(+)$ channel's slow inactivation gate. J Gen Physiol 116, 623-636 (2000).

34. H. P. Larsson, F. Elinder, A conserved glutamate is important for slow inactivation in $\mathrm{K}_{+}$ channels. Neuron 27, 573-583 (2000).

35. A. Mathie, E. Al-Moubarak, E. L. Veale, Gating of two pore domain potassium channels. J Physiol 588, 3149-3156 (2010).

36. M. Lolicato et al., K2P channel C-type gating involves asymmetric selectivity filter orderdisorder transitions. Sci Adv 6 (2020).

37. B. Li, R. A. Rietmeijer, S. G. Brohawn, Structural basis for $\mathrm{pH}$ gating of the two-pore domain $\mathrm{K}(+)$ channel TASK2. Nature 586, 457-462 (2020). 
a
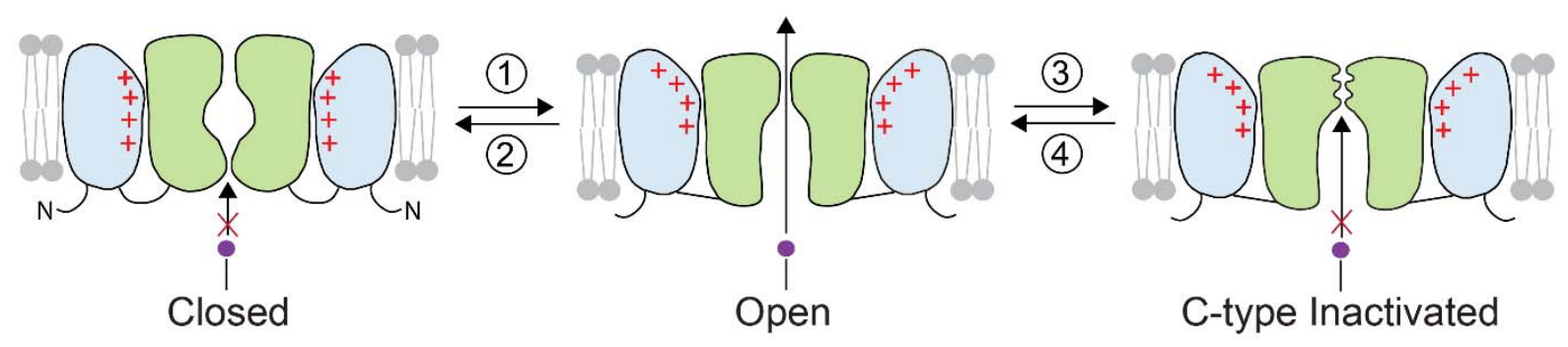

b

C
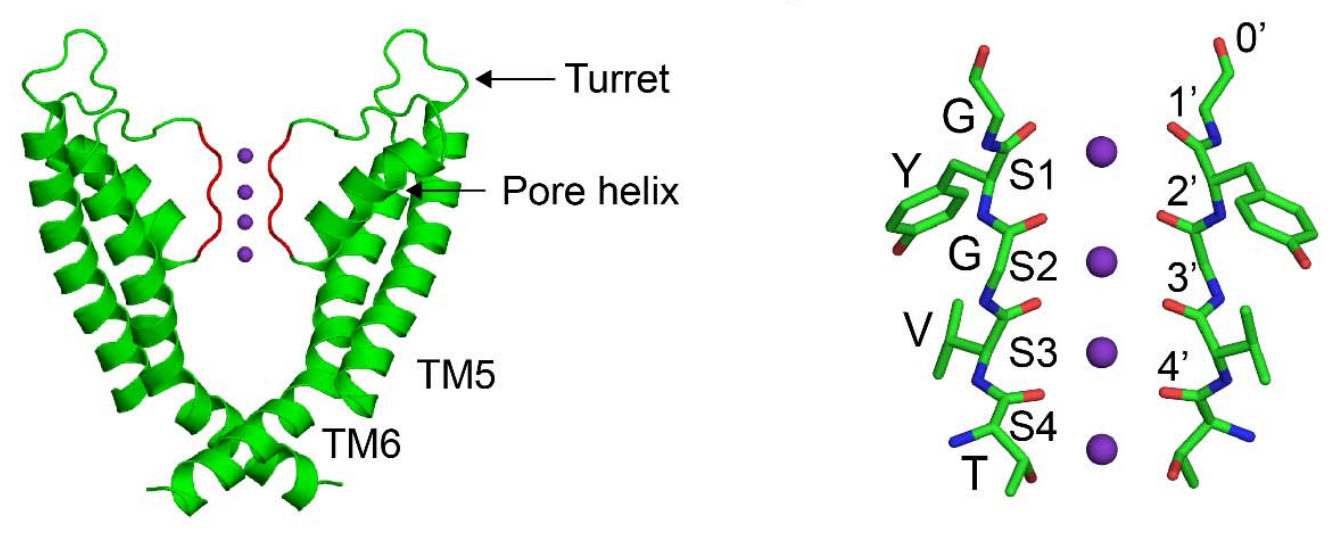

d
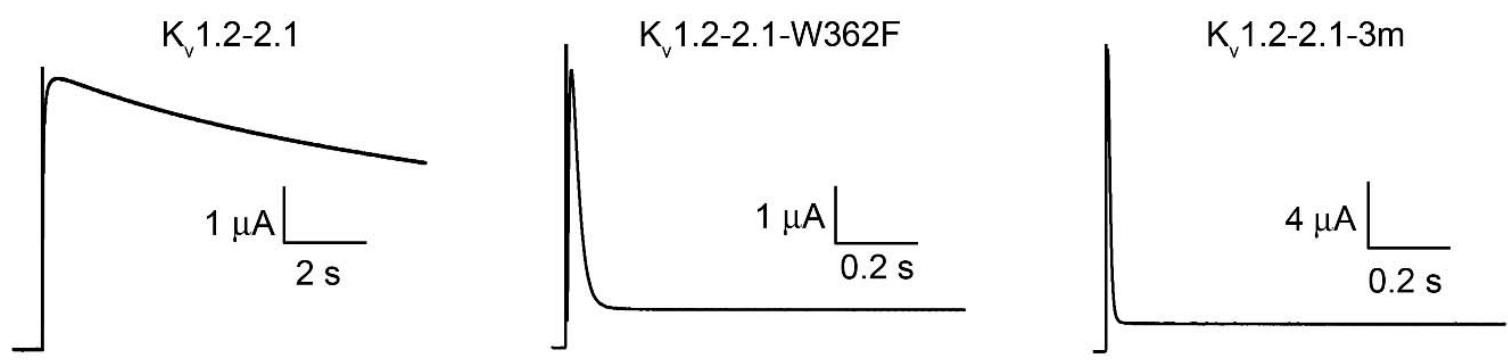

e
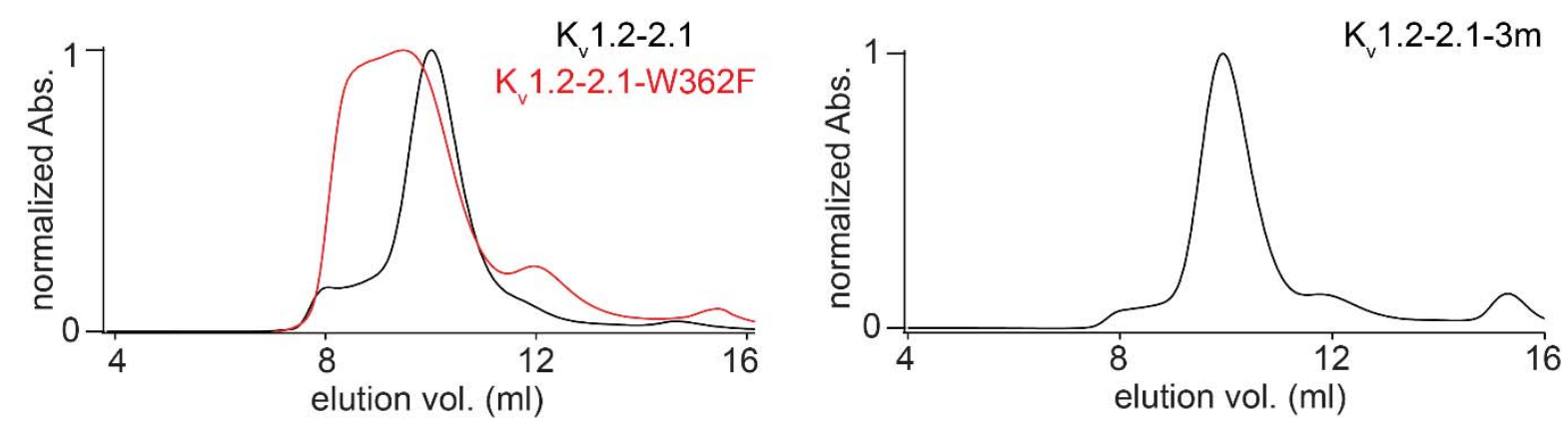

Figure 1: C-type inactivation in the $\mathrm{K}_{\mathrm{v}}$ 1.2-2.1 channel. a) Gating mechanisms in $\mathrm{K}_{\mathrm{v}}$ channels. The gating processes in a $K_{v}$ channel of activation (1), deactivation (2), C-type inactivation (3) and recovery from inactivation (4) are depicted. TM4 in the voltage sensor domain is colored red. Voltage gating involves the movement of the TM4 helix while C-type inactivation involves structural changes at the selectivity filter. b) The pore domain of the $\mathrm{K}_{\mathrm{v}} 1.2$ - 
2.1 channel (pdb: 2r9r). Two opposite subunits are shown, with the selectivity filter colored red and the $\mathrm{K}^{+}$ions bound at the selectivity filter depicted as purple spheres. c) Close-up view of the selectivity filter of the $\mathrm{K}_{\mathrm{v}} 1.2-2.1$ channel. Two opposite subunits are shown in stick representation. The ion-binding sites in the selectivity filter (S1-S4) and the 0'-4' carbonyl bonds are labeled. d) C-type inactivation in the $\mathrm{K}_{\mathrm{v}} 1.2-2.1, \mathrm{~K}_{\mathrm{v}} 1.2-2.1-\mathrm{W} 362 \mathrm{~F}$ and the $\mathrm{K}_{\mathrm{v}} 1.2-2.1$ $3 \mathrm{~m}$ channels. Time course of current elicited by stepping the voltage from $-80 \mathrm{mV}$ holding potential to $40 \mathrm{mV}$ with $100 \mathrm{mM}$ external $\mathrm{K}^{+}$. Initial spikes observed are the capacitance transients. The inactivation time constants (in ms) determined were $11890 \pm 1666( \pm \mathrm{SEM}, \mathrm{n}=$ 4) for $K_{v} 1.2-2.1,22.4 \pm 7.2$ (9) for the $K_{v} 1.2-2.1-W 362 F$ and $5.5 \pm 0.7$ (10) for the $K_{v} 1.2-2.1-3 m$ channels. e) Size exclusion chromatography profiles of the purified $\mathrm{K}_{\mathrm{v}} 1.2-2.1, \mathrm{~K}_{\mathrm{v}} 1.2-2.1-\mathrm{W} 362 \mathrm{~F}$ and the $\mathrm{K}_{\mathrm{v}} 1.2-2.1-3 \mathrm{~m}$ channel. 
a

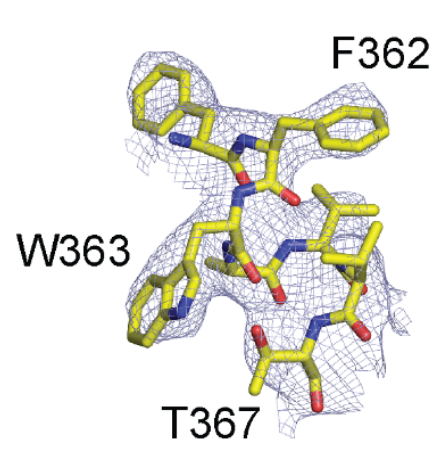

C

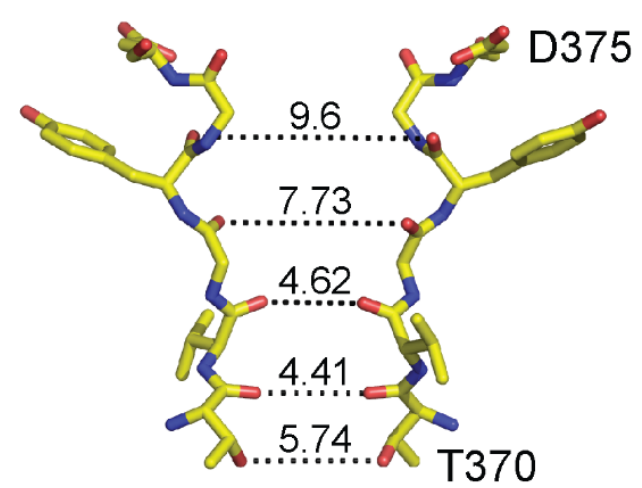

e

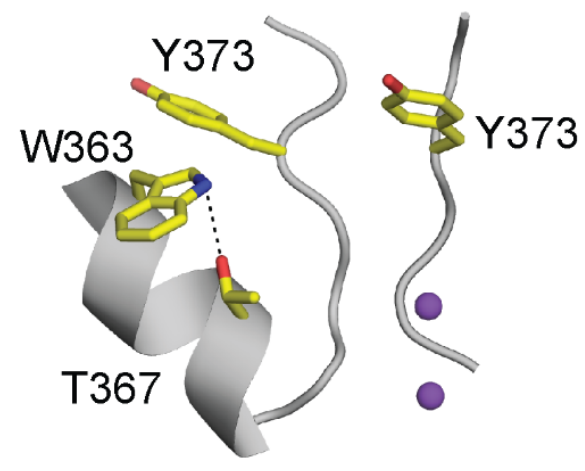

g

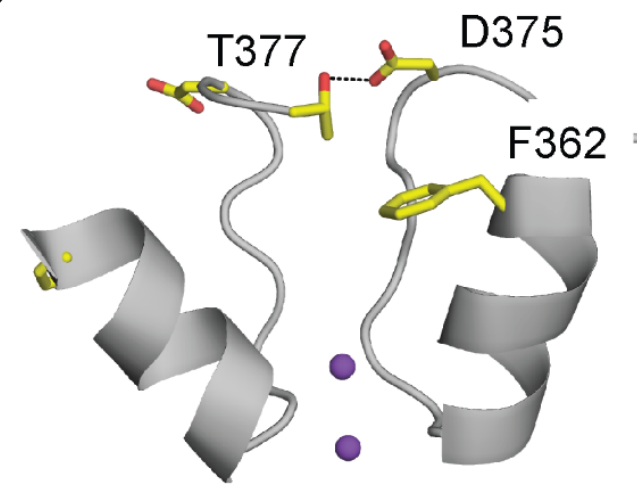

b

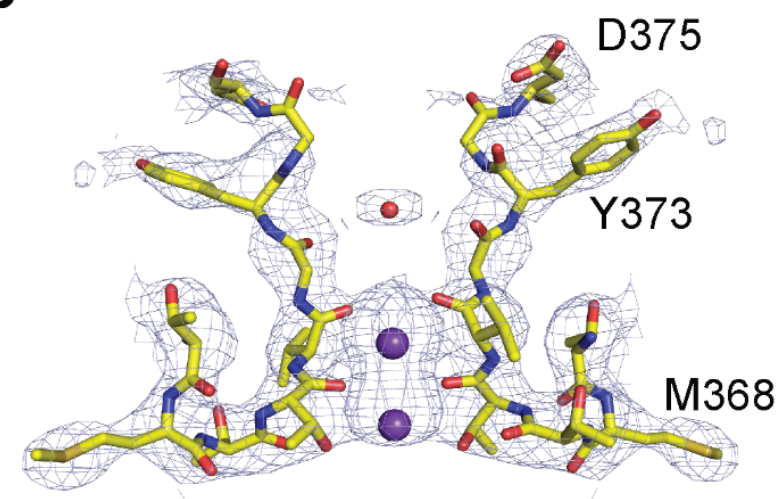

d

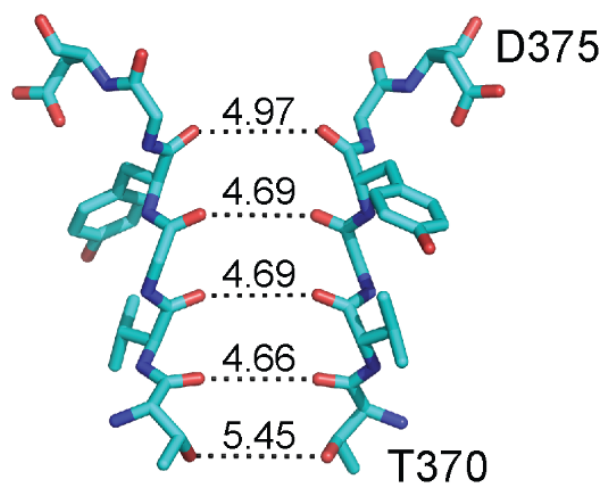

f

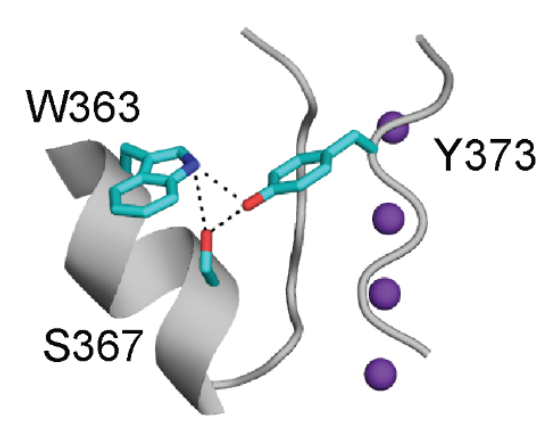

h 
Figure 2: Changes in the selectivity filter on C-type inactivation. a) The pore helix of the $\mathrm{K}_{\mathrm{v}}$ 1.2-2.1-3m channel. The $2 \mathrm{~F}_{\mathrm{o}}-\mathrm{F}_{\mathrm{c}}$ electron density map contoured at $1.4 \sigma$ is shown with residues 361-367 as sticks. The W362F and S367T substitutions present in the $\mathrm{K}_{\mathrm{v}} 1.2-2.1-3 \mathrm{~m}$ channel are indicated. b) The selectivity filter of the $\mathrm{K}_{\mathrm{v}} 1.2-2.1-3 \mathrm{~m}$ channel. The $2 \mathrm{~F}_{\mathrm{o}}-\mathrm{F}_{\mathrm{c}}$ electron density map contoured at $0.9 \sigma$ is shown with residues $368-375$ as sticks, $\mathrm{K}^{+}$ions as purple spheres and water molecule as a red sphere. c,d) Comparison of the selectivity filter of the $\mathrm{K}_{\mathrm{v}} 1.2-2.1-3 \mathrm{~m}$ (c) and the $\mathrm{K}_{\mathrm{v}} 1.2-2.1$ channel (d). Residues 370-375 are shown as sticks. The distance (in $\AA$ ) between the carbonyl oxygens and the Thr370 side chain hydroxyl group in the opposite subunits are indicated by dotted lines. $\mathbf{e , f , g , h ) ~ C l o s e - u p ~ o f ~ t h e ~ s e l e c t i v i t y ~ f i l t e r ~ a n d ~ t h e ~}$ pore helix showing the $\mathrm{Y} 373$ side chain in the $\mathrm{K}_{\mathrm{v}} 1.2-2.1-3 \mathrm{~m}(\mathbf{e})$ and $\mathrm{K}_{\mathrm{v}} 1.2-2.1$ channel $(\mathbf{f})$ and the D375 side chain in the $\mathrm{K}_{\mathrm{v}} 1.2-2.1-3 \mathrm{~m}(\mathbf{g})$ and $\mathrm{K}_{\mathrm{v}} 1.2-2.1$ channel $(\mathbf{h})$. 
a
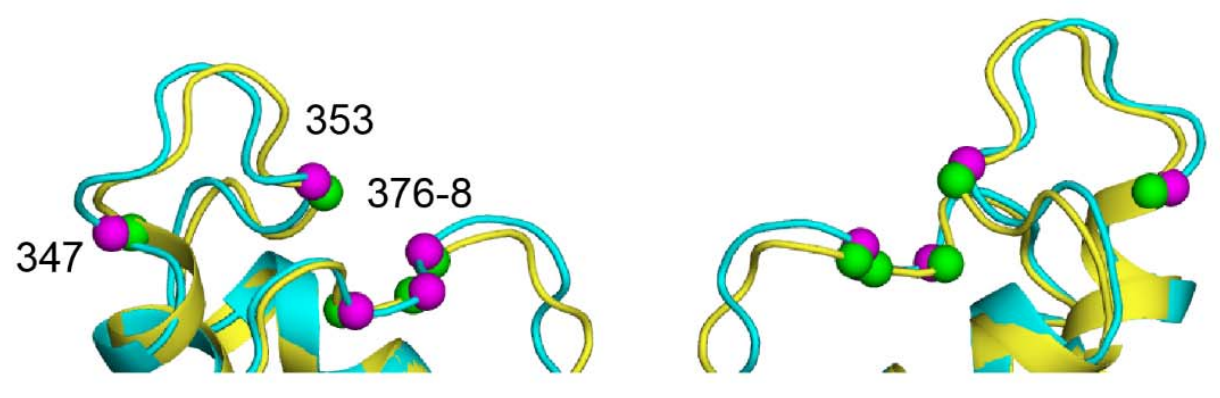

b

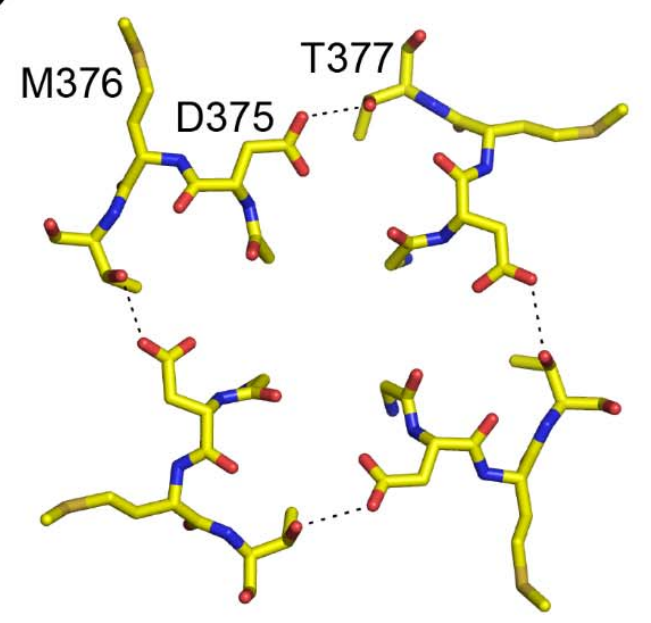

C

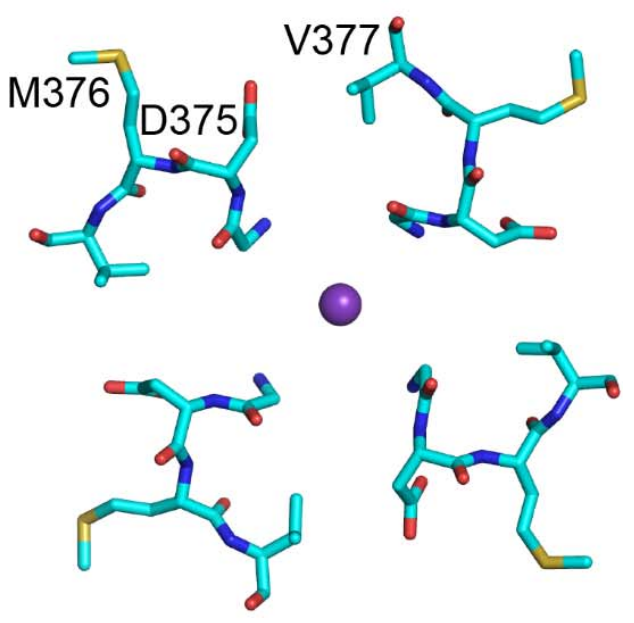

d
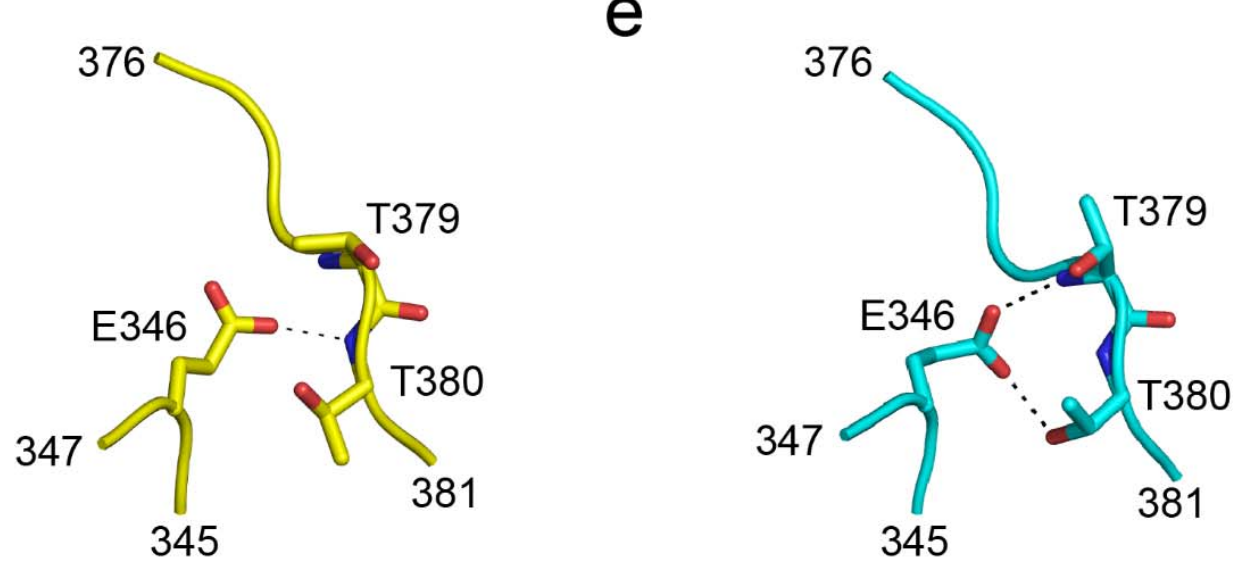

Figure 3: The outer pore region in the C-type inactivated channel. a) Superposition of the outer pore region of the $\mathrm{K}_{\mathrm{v}} 2.1-2.1-3 \mathrm{~m}$ (yellow) and the $\mathrm{K}_{\mathrm{v}} 2.1-2.1$ channel (cyan). Two opposite subunits are shown. Some of the positions at which fluorescence probes introduced detect structural changes on C-type inactivation are indicated (green spheres in $\mathrm{K}_{\mathrm{v}} 2.1-2.1-3 \mathrm{~m}$ and magenta spheres in $\mathrm{K}_{\mathrm{v}} 2.1-2.1$ ). $\left.\mathbf{b}, \mathbf{c}\right)$ Top view of the extracellular mouth of the pore in the $\mathrm{K}_{\mathrm{v}}$ 2.1-2.1-3m (b) and the $\mathrm{K}_{\mathrm{v}} 1.2-2.1$ channel (c) shows changes in conformation of D375 and M376. The $\mathrm{H}$-bond interaction between D375 and T377 in adjacent subunits of the $\mathrm{K}_{\mathrm{v}} 2.1-2.1$ $3 \mathrm{~m}$ channel is shown. d,e) $\mathrm{H}$-bond interactions of E346 in $\mathrm{K}_{\mathrm{v}} 1.2-2.1-3 \mathrm{~m}$ (d) and $\mathrm{K}_{\mathrm{v}} 1.2-2.1$ channel (e) are shown. 
a

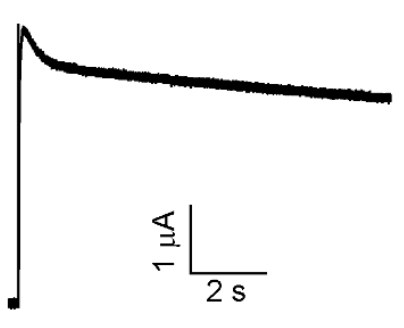

b

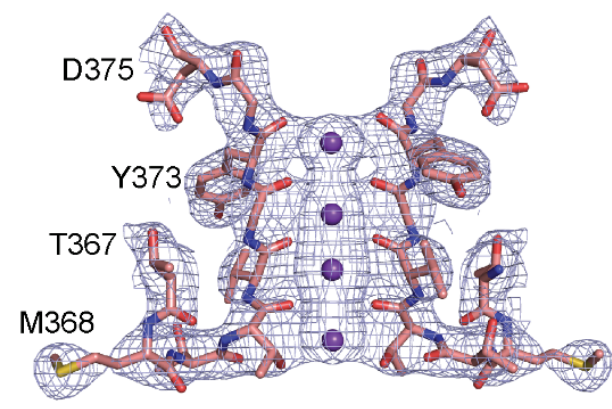

C

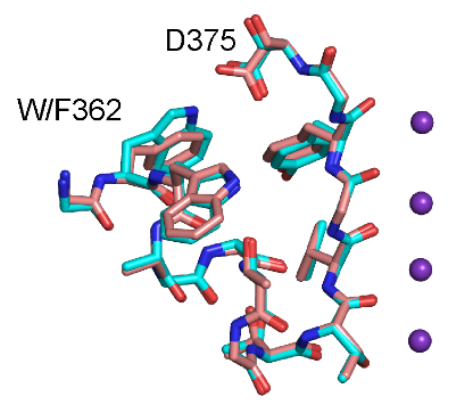

Figure 4: Structure of the selectivity filter in the $\mathrm{K}_{\mathrm{v}} 2.1 \mathrm{ch}-2 \mathrm{~m}$ channel. a) C-type inactivation in the $\mathrm{K}_{\mathrm{v}} 1.2-2.1-2 \mathrm{~m}(\mathrm{~W} 362 \mathrm{~F}, \mathrm{~S} 367 \mathrm{~T})$ channel. Time course of the current elicited by stepping the voltage from $-80 \mathrm{mV}$ holding potential to $40 \mathrm{mV}$ in $100 \mathrm{mM}$ external $\mathrm{K}^{+}$. b) Electron density of the selectivity filter of $K_{v} 1.2-2.1-2 m$. The $2 F_{o}-F_{c}$ electron density map contoured at $1.3 \sigma$ is shown with residues 367-375 as sticks, and the $\mathrm{K}^{+}$ions in the selectivity filter shown as purple spheres. c) Superposition of the pore helix and filter region (residues 362-375) of the $K_{v} 1.2-2.1-$ $2 \mathrm{~m}$ and the $\mathrm{K}_{\mathrm{v}} 1.2-2.1$ channel. 
a

\section{Conductive Inactivated}

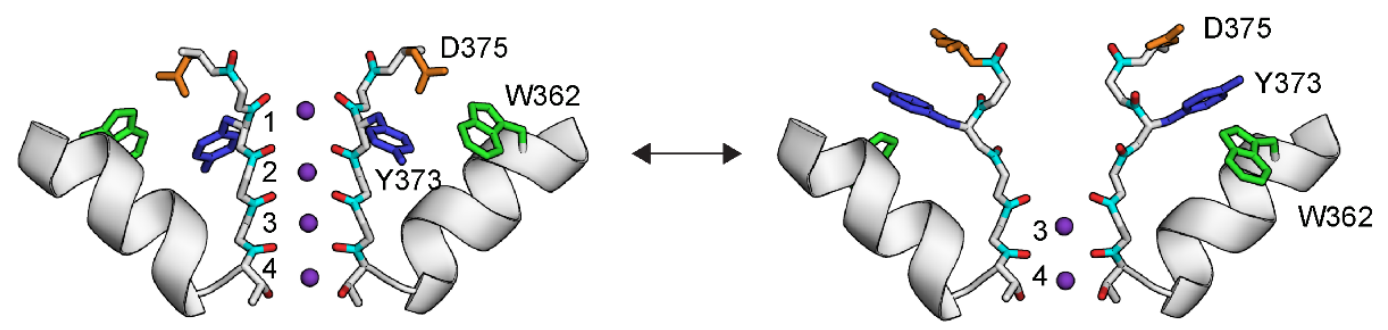

b

Conductive

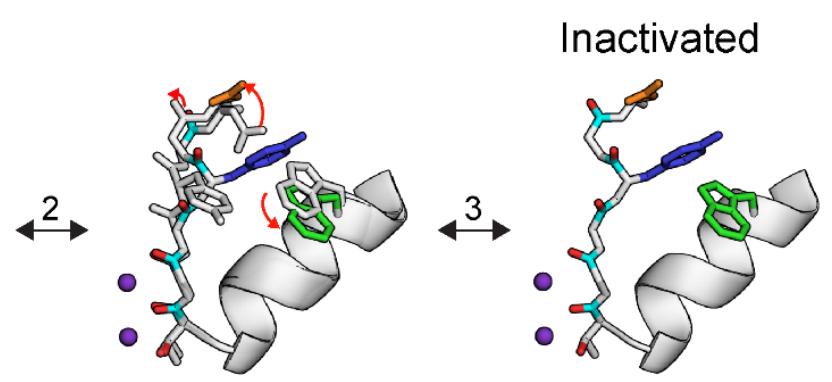

Figure 5: C-type inactivation in a $\mathrm{K}_{\mathrm{v}}$ channel. a) A model for the selectivity filter in the conductive and the $\mathrm{C}$-type inactivated state. The conductive state of the selectivity filter is observed in the $\mathrm{K}_{\mathrm{v}} 1.2-2.1$ channel (pdb: 2r9r) while the C-type inactivated state is based on the structure of the selectivity filter in the $K_{v} 1.2-2.1-3 m$ channel. Two opposite subunits are shown. b) A potential sequence of structural transitions in the selectivity filter during C-type inactivation. Step 1 is the loss of ion binding to the S1 and S2 sites. Step 2 is the switch of the Y373 residue and the flip of the 1' and the 2' carbonyl groups. Step 3 is the rotation of the D375 residue to the extracellular side and the flip of the 0 ' carbonyl group. These transitions result in a dilation of the S1 and S2 ion binding sites in the selectivity filter. One subunit is shown. 